PAPER

\title{
Platelet-leukocyte interaction and platelet activation in migraine: a link to ischemic stroke?
}

\author{
J A Zeller, K Frahm, R Baron, R Stingele, G Deuschl
}

J Neurol Neurosurg Psychiatry 2004;75:984-987. doi: 10.1136/jnnp.2003.019638

See end of article for authors' affiliations ......................

Correspondence to: Dr J A Zeller, Klinikum der Christian Albrechts Universität, Klinik für Neurologie,

Niemannsweg 147, D24105 Kiel, Germany; j.zeller@neurologie. uni-kiel.de

Received 28 May 2003 In revised form 22 October 2003 Accepted 26 November 2003

\begin{abstract}
Objectives: Migraine has been identified as an independent risk factor for ischemic stroke. Both neurogenic inflammation and platelet activation have been linked to the pathophysiology of migraine. Increased platelet activation results in up-regulation of specific binding to leukocytes which promotes proinflammatory leukocyte secretion and their tethering to endothelium, a mechanism that has been demonstrated in stroke and which could provide a link to migraine. We aimed to determine whether platelet-leukocyte aggregation is increased in migraine patients outside an acute attack.

Methods: Seventy two patients with migraine according to IHS criteria were compared to a control group $(n=72)$. Whole blood flow cytometry was used to quantify the activation dependent $P$ selectin on the platelet, and to assess the fraction of platelets bound to the different leukocyte subsets.

Results: Migraine patients showed significantly more platelet-leukocyte aggregates compared to the control subjects $(p=0.003)$. This effect was driven by an increased polymorphonuclear cell-platelet aggregation $(p=0.003)$ whereas platelet aggregation with monocytes and lymphocytes was not. Platelet activation was also increased $(p=0.001)$.

Conclusions: In migraine pro-inflammatory platelet adhesion to leukocytes occurs during the headache free interval similar to that seen in acute coronary and cerebrovascular syndromes. This may suggest a link between migraine and stroke on a cellular level.
\end{abstract}

1 n numerous epidemiological studies, migraine has been identified as an independent risk factor for cerebral ischaemia, particularly in young females (see Crassard ${ }^{1}$ for a review). A mechanism connecting the two disease entities has not yet been identified. For many years platelet dysfunction has been linked to the pathophysiology of migraine. Several investigators found indirect evidence for increased platelet activation during migraine attacks and the headache free period (see Kitano ${ }^{2}$ for reference). In addition, aggregometric studies suggested decreased platelet responsiveness after ex vivo stimulation, ${ }^{3}{ }^{4}$ which has been attributed to an "exhaustion" of platelets following in vivo stimulation.

Platelet activation not only propagates mutual and endothelial adhesion and aggregation but also plays an important role in up-regulating inflammatory processes by interacting with leukocytes, especially monocytes and neutrophils. ${ }^{5-7}$ The intercellular binding is mediated mainly between the platelets' activation dependent P selectin epitope and the $\mathrm{P}$ selectin glycoprotein ligand 1 (PSGL-1) on the leukocyte. ${ }^{8}$ This results in increased leukocyte superoxide anion and tumour necrosis factor production, enhanced endothelial adhesion and transendothelial migration, but also increased platelet mediated aggregation and thromboxane release. ${ }^{9}$ The important role of increased platelet activation and platelet-leukocyte interaction in the development of atherothrombotic disease has been reported in thrombocythemic patients, ${ }^{10}$ in the pathophysiology of ischemic stroke, ${ }^{11}{ }^{12}$ and in symptomatic coronary disease. ${ }^{13-16}$

We hypothesised that if increased platelet activation and platelet-leukocyte interaction can also be measured in migraine patients during the headache free interval, this parallel with stroke patients would highlight a possible common pathophysiological pathway in both diseases, which may contribute to the epidemiological coincidence. Although the pathophysiology of migraine is known to include neurogenic inflammation with significant intercellular signalling, the cellular interaction between leukocytes and platelets in migraine has not been investigated.

To test our hypothesis we used flow cytometry to directly test on a cellular level whether migraine patients show upregulated platelet leukocyte interaction in addition to increased baseline platelet activation.

\section{METHODS \\ Patients}

The study group consisted of 72 patients who sequentially attended the Kiel headache outpatient clinic with confirmed diagnosis of migraine according to IHS criteria ${ }^{17}$ and were willing to give written informed consent. The study was approved by the university's ethics committee. Forty eight patients suffered from migraine without aura and 24 from migraine with aura; none of the patients had a history of myocardial infarction or stroke. The mean age of migraine patients was 45.6 years (standard deviation (SD) 12.4). Fifty nine patients (82\%) were female, and of these $36(61 \%)$ were premenopausal. The mean age in the control group was 43.9 years (SD 18.3) and 56\% were female. In the patient group nobody reported a history of cerebral ischaemia, and one patient had one episode of suspected angina without cardiac findings in the subsequent diagnostic investigation. Three patients mentioned intermittent cardiac arrhythmia which needed no specific treatment. Two patients were diabetic without insulin treatment. Seven of the patients were smoking regularly as opposed to six members of the control group. At the time of blood sampling, 29 patients (40.3\%) were taking $\beta$ blockers as a single prophylactic agent, 13 (18\%) were on $\beta$ blockers and amitriptyline, three on a combination of $\beta$ blocker and cyclandelat, and two on cyclandelat only. Twenty four patients (33.3\%) were taking no medication at all. One case remained unclear with regard to drug prophylaxis. The control group consisted of volunteers from medical staff and patients who were all free from migraine or other forms of chronic or remitting headache, 
history of cardiac or cerebrovascular disease, diabetes, and acute infection. Both patients and controls had been free from acetylsalicylic acid or other non steroidal antiflammatory drugs for at least 2 weeks, and nobody in the control group was taking tricyclic antidepressants.

\section{Methods}

Venous blood was drawn from an antecubital vein between 10 a.m. and 1 p.m., anticoagulated with $3.8 \%$ sodium citrate and processed after 10 min of resting time without further manipulation. Blood samples were kept at body temperature at all times. For the flow cytometric assays, we used direct fluorescent markers (all commercially available; Coulter Immunotech, Krefeld, Germany). Whole blood was diluted 1:10 with warmed HEPES buffer and two aliquots of $50 \mu \mathrm{l}$ were incubated with CD 61-PE (an activation independent subunit of the GP IIb/IIIa complex) to immunologically identify all platelets. Simultaneously, in a one step procedure, the sample for measuring platelet activation was stained with anti CD 62-P. The other sample was double stained with the panleukocytic marker CD 45 to identify leukocytes. After incubation for $5 \mathrm{~min}$, the process was stopped using cold buffer, immediately followed by flow cytometry.

Platelets were identified by their size and granulation properties using forward/sideward scatter and staining with the panthrombocytic marker CD 61-PE. By double gating of CD 45 positivity, forward and sideward scatter properties we discriminated between leukocyte subsets. Then the double fluorescent particles positive for both the leukocytic CD 45 and platelet CD 61 epitope were counted as plateletleukocyte aggregates and platelet-leukocyte subset aggregates, respectively. Since red cells are not specifically stained and unspecific fluorescence is far below detection threshold, it is not necessary in this assay to perform red cell lysis, thus avoiding further artefacts. Measurements were performed with an Epics XL cytometer (Coulter Immunotech, Krefeld, Germany). This assay is an established procedure in various academic institutions in our country and has been in routine use in our laboratory for the 3 years prior to this investigation.

\section{Statistical analysis}

All values are expressed as means (SD). The Mann-Whitney $\mathrm{U}$ test was used to compare groups, and $\mathrm{p}<0.05$ was considered significant in two tailed tests.

\section{RESULTS}

A total of 72 migraine patients ( 59 women and 13 men) were included in this study. Two thirds of the patients suffered from migraine without aura and one third from migraine with aura, a ratio commonly seen in the distribution of migraine subtypes in specialised clinics. Within the patient group there were no significant differences regarding age or sex distribution. Since we deliberately examined migraine patients outside an acute attack, none of the subjects had experienced headaches in the $24 \mathrm{~h}$ prior to blood sampling. Nineteen of the 72 patients (26\%) reported a migraine attack 1-3 days prior to the investigation, and 17 had used an oral or subcutaneous triptane. Recent migraine attack and triptane intake did not differ statistically between both migraine groups. In the control group (as in control groups in previous studies), there was no gender difference regarding platelet-leukocyte adhesion or platelet activation. Thus we regarded the gender mismatch between controls and migraine patients as insignificant.

\section{Platelet-leukocyte aggregation}

Migraine patients showed a significantly increased number of platelet-leukocyte aggregates $(5.70 \%$ v $3.89 \%$ in controls, $\mathrm{p}=0.003)$. This was due to the increased proportion of platelets aggregating with polymorphonuclear cells $(7.26 \% \mathrm{v}$ $4.98 \%, p=0.002$; table 1). Aggregation of platelets with monocytes and lymphocytes was, although raised, not significantly increased in this sample size. Analysis of age dependency (two groups above and below the 50th percentile) and of the influence of prophylactic medication (47 with, 24 without) revealed no statistical difference.

\section{Platelet activation}

During the headache free interval investigated here, migraine patients expressed more of the activation dependent platelet epitope $\mathrm{P}$ selectin compared to the control subjects (1.41 $(0.22)$ arbitrary units, $p=0.001$; table 1$)$. This was mainly influenced by the highly significant ( $1.42(0.21), p<0.001)$ difference in patients suffering from migraine without aura; in those affected by migraine with aura the difference failed to reach significance in our sample (1.39 (0.23), $\mathrm{p}=0.082)$. As seen with aggregation, values were not age or drug dependent.

\section{DISCUSSION}

This study represents the first investigation of intercellular communication between platelets and leukocytes in migraine patients. The hypothesised significant increase in intercellular platelet-leukocyte interaction does exist in migraine patients similar to that seen in many patients with acute or early post-acute cerebral ischaemia. In addition, those without aura have a higher baseline of platelet activation compared to controls.

Platelet activation has been investigated before by measuring plasma levels of the platelet secretion products or urinary platelet metabolites. ${ }^{218-20}$ Using a photometric technique, whole blood flow cytometry, the externalisation of activation dependent platelet epitopes can be quantified on a cell to cell level in an automated procedure. In this way, dilution effects in the whole body plasma compartment and possible artefacts by metabolic processes can be avoided. We use P selectin antibodies which quantify the surface expression of the CD 62P epitope (P selectin) on the platelet. This alpha granule protein is externalised on to the platelet surface during platelet activation. The epitope has been shown to be a reliable activation marker in cardiac and neurovascular patients. ${ }^{11} 1621$ Also, direct detection of both leukocyte and platelet specific cell properties allows immediate ex vivo identification of aggregates without further artefact prone immunostaining and microscopy.

Cytometric measurement of activation dependent platelet epitopes aims directly at the platelets' cellular surface with very little ex vivo manipulation, and is less prone to artefacts.

Table 1 Platelet-leukocyte aggregates (percent of platelet population (SD)) and platelet activation (mean fluorescence in arbitrary units(SD))

\begin{tabular}{llll}
\hline & $\begin{array}{l}\text { Controls, } \\
\mathrm{n}=\mathbf{7 2}(\mathrm{SD})\end{array}$ & $\begin{array}{l}\text { All migraine, } \\
\mathrm{n}=\mathbf{7 2}(\mathrm{SD})\end{array}$ & $\begin{array}{l}\mathrm{p} \text { Value } \mathbf{v} \text { controls, } \\
\mathrm{n}=\mathbf{4 8}(\mathrm{SD})\end{array}$ \\
\hline $\begin{array}{l}\text { Leukocyte-platelet } \\
\text { aggregation }\end{array}$ & $3.89(2.19)$ & $5.70(4.61)$ & 0.003 \\
$\begin{array}{l}\text { Polymorph-platelet } \\
\text { aggregation }\end{array}$ & $4.98(3.10)$ & $7.26(6.83)$ & 0.003 \\
$\begin{array}{l}\text { Monocyte-platelet } \\
\text { aggregation }\end{array}$ & $5.57(4.21)$ & $6.55(5.45)$ & 0.201 \\
$\begin{array}{l}\text { Lymphocyte-platelet } \\
\text { aggregation }\end{array}$ & $0.58(0.67)$ & $0.77(0.76)$ & 0.075 \\
$\begin{array}{l}\text { Mean platelet } \\
\text { activation }\end{array}$ & $1.29(0.18)$ & $1.41(0.22)$ & 0.001 \\
\hline
\end{tabular}


The observation of augmented platelet activation in migraine patients confirms previous reports that used indirect methods. We suggest that enhanced platelet activation in migraine patients is a marker of the inflammatory process in the trigeminovascular system and cellular interaction rather than a precipitating factor in migraine pathogenesis.

As regards isolated lymphocyte properties, altered white cell function in migraine has been reported: both a significant rise in $\mathrm{CD} 3+$ ( $\mathrm{T}$ lymphocyte) $\mathrm{CD} 16+56+$ (natural killer lymphocytes) classified lymphocytes in migraine without aura patients outside an attack were seen ${ }^{22}$ as well as raised B lymphocyte counts and decreased CD8 (T suppresser) positive lymphocytes in migraine patients when compared to controls. ${ }^{23}$ Empl et al ${ }^{24}$ described an increased CD4 ( $\mathrm{T}$ helper) count in patients with migraine with or without aura compared to controls. In addition they found significantly higher proportions of integrin high expressing $\mathrm{T}$ helper cells in subjects with migraine without aura compared to migraine with aura.

Using this method, our results do not support lymphocytic reaction with memory cells, which represents a longer standing process of inflammation indicative of immunological activation in migraine patients, but acute phase leukocyte subsets (as seen by the increased neutrophil fraction attached to platelets). This suggests that even during the quiescent phase increased inflammatory signalling occurs. This may involve kinins like interferons and tumour necrosis factor and soluble adhesion molecules ${ }^{25}$ which lead in a partly selfpropagating process to direct intercellular interaction among different subsets of leukocytes and between leukocytes and platelets.

Increased platelet-leukocyte adhesion has also been described in patients at risk of reocclusion after coronary stenting ${ }^{13}$ and in patients with unstable angina ${ }^{14}$; this could be reduced by blockage of the activation dependent platelet GP IIb/IIIa receptor. ${ }^{15}$

This observation of increased intercellular communication in both cardiovascular disease and migraine may provide a direct link to ischemic stroke for which migraine has been proven to be an independent risk factor, particularly in premenopausal women. ${ }^{26-32}$ In a recent review of migraine, haemostasis, and ischemic stroke in young women, ${ }^{1}$ the authors found it impossible to conclude from previous trials how migraine and cerebral ischaemia are connected because of contradicting results and conflicting techniques. Likewise, studies on the incidence of antiphospholipid antibodies or prothrombotic genetic risk factors (mainly factor V Leiden and prothrombin mutation) in migraine patients were not promising, particularly since the latter are known to mainly affect venous system thrombosis, hence not a platelet initiated mechanism. However, it seems reasonable to study risk factors for increased platelet aggregability in the light of previous reports of patients with essential thrombocythaemia and migraine whose symptoms were reduced by antiplatelet therapy. ${ }^{33}$ Additional data suggest a preventive effect of aspirin alone or in combination with dipyridamole on migraine recurrence. ${ }^{35-37}$

We strongly support this approach: patients with acute ischemic stroke of large vessel but not of cardiac origin show increased platelet activation ${ }^{11}$ and, particularly following infections, increased leukocyte-platelet aggregates. ${ }^{12}$ The role of this intercellular communication is still subject to research: possible explanations include a causative model where infection via platelet and leukocyte activation triggers thrombus formation as well as post-thrombotic processes where platelet stimulated leukocyte activation adds to excitotoxic substance release. Increased platelet activation in essential thrombocythaemia leading to stroke has also been reported. ${ }^{38}$ These coincidences of altered platelet function in migraine patients, as reported here, and those suffering from stroke, myocardial infarction, and thrombocythaemia are promising links that should be further pursued. Although primary platelet dysfunction as an initiator for migraine is unlikely, the increased activation and leukocyte, namely neutrophil, aggregation constitute a prothrombotic risk that eventually may lead to ischemic stroke. It must also be considered that inflammation and infection itself have been proven to be independent risk factors of ischemic stroke. ${ }^{39-41}$

In migraine pro-inflammatory platelet adhesion to leukocytes occurs during the headache free interval similar to that seen in acute coronary and cerebrovascular syndromes. This may suggest a link between migraine and stroke on a cellular level. Studies with correlation of this haemostasiological parameter to other indicators of altered coagulation biochemistry in migraine patients and comparisons between migraine patients with and without stroke, and subgroup analysis of data from stroke prevention trials on the efficacy of antiplatelet therapy in migraine patients will aid the understanding of underlying mechanisms and the deduction of possible prophylactic concepts.

\section{Authors' affiliations}

J A Zeller, K Frahm, R Baron, R Stingele, G Deuschl, Department of Neurology, Christian Albrechts University Kiel, Kiel, Germany

Competing interests: none declared

\section{REFERENCES}

1 Crassard I, Conard J, Bousser MG. Migraine and haemostasis. Cephalalgia 2001;21:630-6.

2 Kitano A, Shimomura T, Takeshima T, et al. Increased 11dehydrothromboxane B2 in migraine: platelet hyperfunction in patients with migraine during headache-free period. Headache 1994;34:515-8.

3 Tozzi-Ciancarelli MG, De Matteis G, Di Massimo C, et al. Oxidative stress and platelet responsiveness in migraine. Cephalalgia 1997; 17:580-4.

4 Joseph R, Welch KM, D'Andrea G. Serotonergic hypofunction in migraine: a synthesis of evidence based on platelet dense body dysfunction. Cephalalgia 1989;9:293-9.

5 Nagata K, Tsuji T, Todoroki N, et al. Activated platelets induce superoxide anion release by monocytes and neutrophils through P-selectin (CD62). $\mathrm{J}$ Immunol 1993;151:3267-73.

6 Weyrich AS, Elstad MR, McEver RP, et al. Activated platelets signal chemokine synthesis by human monocytes. J Clin Invest 1996;97:1525-34.

7 Nash GB. Adhesion between neutrophils and platelets: a modulator of thrombotic and inflammatory events? Thromb Res 1994;74:S3-11.

8 McEver RP, Cummings RD. Role of PSGL-1 binding to selectins in leukocyte recruitment. J Clin Invest 1997; 100:S97-103.

9 Faraday N, Scharpf RB, Doddo JM, et al. Leukocytes can enhance plateletmediated aggregation and thromboxane release via interaction of $\mathrm{P}$-selectin glycoprotein ligand 1 with P-selectin. Anesthesiology 2001;94:145-51.

10 Falanga A, Marchetti $M$, Evangelista V, et al. Polymorphonuclear leukocyte activation and hemostasis in patients with essential thrombocythemia and polycythemia vera. Blood 2000;96:4261-6.

11 Zeller JA, Tschoepe D, Kessler C. Circulating platelets show increased activation in patients with acute cerebral ischemia. Thromb Haemost 1999;81:373-7.

12 Zeller JA, Zunker P, Lenz A, et al. Clinical application of detecting platelet activation markers in cerebrovascular disease. Infus Ther Transfus Med 2000;27:250-5.

13 Mickelson JK, Lakkis NM, Villarreal-Levy G, et al. Leukocyte activation with platelet adhesion after coronary angioplasty: a mechanism for recurrent disease? J Am Coll Cardiol 1996;28:345-53.

14 OH I, Neumann FJ, Gawaz M, et al. Increased neutrophil-platelet adhesion in patients with unstable angina. Circulation 1996;94:1239-46.

15 Neumann FJ, Zohlnhofer D, Fakhoury L, et al. Effect of glycoprotein Ilb/Illa receptor blockade on platelet-leukocyte interaction and surface expression of the leukocyte integrin Mac-1 in acute myocardial infarction. J Am Coll Cardiol 1999;34:1420-6.

16 Gawaz M, Neumann FJ, OH I, et al. Platelet function in acute myocardial infarction treated with direct angioplasty. Circulation 1996;93:229-37.

17 Headache Classification Committee of the International Headache Society Classification and diagnostic criteria for headache disorders, cranial neuralgias and facial pain. Cephalalgia 1988;8:1-96.

18 Puig-Parellada P, Planas JM, Gimenez J, et al. Plasma and saliva levels of $P G \mid 2$ and TXA2 in the headache-free period of classical migraine patients. The effects of nicardipine. Headache 1991;31:156-8.

19 Takeshima T, Shimomura T, Takahashi K. Platelet activation in muscle contraction headache and migraine. Cephalalgia 1987;7:239-43. 
20 D'Andrea G Canazi A, Toldo $M$, et al. Drugs and platelet activation in migraine and transient ischemic attacks. Cephalalgia 1985;5(suppl 2):103-8.

21 Tschoepe D, Schultheiss HP, Kolarov P, et al. Platelet membrane activation markers are predictive for increased risk of acute ischemic events after PTCA. Circulation 1993:88:37-42.

22 Mosnaim AD, Kulaga $H$, Adams AJ, et al. Flow cytometric analysis of lymphocyte subsets in migraine patients during and outside of an acute headache attack. Cephalalgia 1998;18:197-201.

23 Leone $M$, Biffi $M$, Leoni $F$, et al. Leukocyte subsets and cortisol serum levels in patients with migraine without aura and chronic tension-type headache. Cephalalgia 1994; 14:139-42.

24 Empl M, Sostak P, Breckner M, et al. T-cell subsets and expression of integrins in peripheral blood of patients with migraine. Cephalalgia 1999; 19:713-7

25 Martelletti P, Stirparo G, Morrone S, et al. Inhibition of intercellular adhesion molecule-1 (ICAM-1), soluble ICAM-1 and interleukin- 4 by nitric oxide expression in migraine patients. J Mol Med 1997;75:448-53.

26 Tzourio C, Tehindrazanarivelo A, Iglesias S, et al. Case-control study of migraine and risk of ischaemic stroke in young women. $B M J$ 1995:310:830-3.

27 Carolei A, Marini C, De Matteis G. History of migraine and risk of cerebral ischaemia in young adults. The Italian National Research Council Study Group on Stroke in the Young. Lancet 1996;347:1503-6.

28 Chang CL, Donaghy M, Poulter N. Migraine and stroke in young women case-control study. The World Health Organisation Collaborative Study of Cardiovascular Disease and Steroid Hormone Contraception. BMJ 1999;318:13-8.

29 Milhaud D, Bogousslavsky J, van Melle G, et al. Ischemic stroke and active migraine. Neurology 2001;57:1805-11.
30 Merikangas KR, Fenton BT, Cheng SH, et al. Association between migraine and stroke in a large-scale epidemiological study of the United States. Arch Neurol 1997;54:362-8

31 Hillbom M, Haapaniemi H, Juvela S, et al. Recent alcohol consumption, cigarette smoking, and cerebral infarction in young adults. Stroke 1995;26:40-5.

32 Bousser MG. Stroke in women: the 1997 Paul Dudley White International Lecture. Circulation 1999;99:463-7.

33 Bousser MG, Conard J, Lecrubier C, et al. Migraine or transient ischemic attacks in a patient with essential thrombocythaemia. Treatment with ticlopidine. Ann Med Interne 1980;131:87-90.

34 Peatfield RC. Can transient ischaemic attacks and classical migraine always be distinguished? Headache 1987;27:240-3.

35 Buring JE, Peto $\mathrm{R}$, Hennekens $\mathrm{CH}$. Low-dose aspirin for migraine prophylaxis. JAMA 1990;264:1711-3.

36 Baldrati A, Cortelli P, Procaccianti G, et al. Propranolol and acetylsalicylic acid in migraine prophylaxis. Double-blind crossover study. Acta Neurol Scand 1983;67:181-6.

37 Ryan RE Sr, Ryan RE Jr. The use of platelet inhibitors in migraine. Adv Neurol 1982:33:247-52.

38 Zeller JA, Allardt A, Deuschl G. Therapy guidance using flow cyłometric platelet function testing in a patient with thrombocytosis and a progressing carotid stenosis (abstract). Cerebrovasc Dis 2001;11(suppl 4):70.

39 Grau AJ, Buggle F, Steichen-Wiehn C, et al. Clinical and biochemical analysis in infection-associated stroke. Stroke 1995;26:1520-6.

40 Bova IY, Bornstein NM, Korczyn AD. Acute infection as a risk factor for ischemic stroke. Stroke 1996;27:2204-6.

41 Macko RF, Ameriso SF, Barndt R, et al. Precipitants of brain infarction. Roles of preceding infection/inflammation and recent psychological stress. Stroke 1996;27:1999-2004.

\section{HISTORICAL NOTE}

\section{Migraine treated by Shakespeare's son-in-law, Dr John Hall}

doi: 10.1136/innp.2004.035634

$\mathrm{T}$ he name of Dr John Hall is familiar to students of Shakespeare, but less known by medical biographers. He was born c. 1575 and died in 1635, ${ }^{1}$ an eminent Stratford physician and herbalist. ${ }^{23}$

Early descriptions of migraine in Greco Roman times are well known. ${ }^{4}$ In De Capitis Passione, Caelius Aurelianus, born in $\mathrm{AD} 400$ in Algeria, described hemicrania, although the term is not encountered in English texts until 1597 (OED). After Caelius Aurelianus little was written until 1672 in Thomas Willis's Two discourses concerning the soul of brutes (De anima brutorum), ${ }^{5}$ where he distinguished different headache types.

An interesting account of migraine and its treatment in his time is included in Hall's much earlier notebook published in 1644, as Select observations on English bodies, or cures both empericall and historicall performed upon very eminent persons in desperate diseases ${ }^{1}$ :

"Observation XXIII. Good-Wife Bessie aged 40, who once a month (yea sometimes twice or thrice) was grievously pained on the right side of her Head, which often ended with vomiting, and in her Fit could neither walk nor stand: was cured thus: First, she took this Vomit; R/the vomiting Infusion ozs $\mathrm{i}$. This wrought six times. For the next day was provided the following pills: R/Pil de Succin. drachms ii. Cephal Fernel. Drachms i. F. Pil N xv. She took three of them before supper, every day till they were spent. After I cause a Vein to be opened to ozs vi. After she took the Decoction: R/Sarsaparilla ozs iv. Water lbx. being sliced, let them infuse for twenty four hours, after boyl them till half be wasted, strain it. Dose a draught morning and night, when she went to bed. For ordinary Drink, she took the second Decoction, which was made of the same Wood, adding lbxv of Water, boyling it without infusion till the third part be wasted".

How much the contribution of Hall influenced the medical content of Shakespeare's writing remains a fascinating source of conjecture. ${ }^{6}$

J M S Pearce

304 Beverley Road, Analby, Hull HU10 7BG, UK; jmspearce@freenet.co.uk

\section{References}

1 Hall J. Select observations on English bodies, or cures both empericall and historicall performed upon very eminent persons in desperate diseases. First published by James Cooke 1644. See: Joseph, Harriet. Shakespeare's son-inlaw; John Hall, man and physician; with a facsimile of the 2nd edn of Hall's "Select Observations on English Bodies". Hamden, Connecticut: Archon Books, 1964

2 Dr John Hall-a short biography. Stratford: The Shakespeare Birthplace Trust [Display].

3 Jones VA. John Hall: seventeenth-century physician of Stratford upon Avon. Proc R Soc Med 1977;70:709-14.

4 Pearce JMS. Historical aspects of migraine. J Neurol Neurosurg Psychiatry 1986:49:1097-1 103.

5 Willis T. Two discourses concerning the soul of brutes (De anima brutorum) Part 1. 1672. Trans S. Pordage. London: Thomas Dring, 1683.

6 Pearce JMS. Dr John Hall and Shakespeare's medicine. J Medical Biography 2004 (in press) 